\title{
A new model for the Warm Absorber in NGC 3783: a single medium in total pressure equilibrium
}

\author{
A. C. Gonçalves ${ }^{1,2}$, S. Collin ${ }^{1}$, A.-M. Dumont ${ }^{1}$, M. Mouchet ${ }^{1,3}$, A. Różańska ${ }^{4}$, L. Chevallier ${ }^{1,4}$, and R. W. Goosmann ${ }^{1}$ \\ ${ }^{1}$ LUTH, Observatoire de Paris-Meudon, 5 Place Jules Janssen, 92195 Meudon Cedex, France \\ e-mail: anabela.goncalves@obspm.fr \\ 2 CAAUL, Observatório Astronómico de Lisboa, Tapada da Ajuda, 1349-018 Lisboa, Portugal \\ 3 APC, Université Denis Diderot Paris 7, 75005 Paris, France \\ ${ }^{4}$ Nicolaus Copernicus Astronomical Center, Bartycka 18, 00-716 Warszawa, Poland
}

Received 11 January 2006 / Accepted 14 March 2006

\begin{abstract}
Context. Many active galactic nuclei exhibit X-ray features typical of the highly ionized gas called "Warm Absorber" (WA). Such a material appears to be stratified, displaying zones of different density, temperature, and ionization. In this paper, we investigate the possibility of modelling the WA gas in NGC 3783 as a single medium in total pressure equilibrium.

Aims. Our goal is to demonstrate that the WA can be well modelled assuming constant total pressure, in contrast to the current descriptions that are based on the presence of multiple regions, each in constant density. The assumption of total pressure equilibrium yields a more physical description of the WA, resulting in the natural stratification of the ionized gas, and providing an explanation for the presence of lines from different ionization states, as observed in WA spectra.

Methods. We have used the photoionization code TITAN, developed by our team, to compute a grid of constant total pressure models with the purpose of fitting the WA in NGC 3783. We have compared our models to the 900 ks Chandra spectrum of NGC 3783 and to previous studies where the WA was described by multiple zones of constant density.

Results. In the case of NGC 3783, the WA features can be well reproduced by a clumpy, ionized gas with cosmic abondances, ionization parameter $\xi=2500 \mathrm{erg} \mathrm{cm} \mathrm{s}{ }^{-1}$, column density $N_{\mathrm{H}}=4 \times 10^{22} \mathrm{~cm}^{-2}$, and constant total pressure.

Conclusions. We have shown that the WA in NGC 3783 can be modelled by a single medium in total pressure equilibrium; this is probably the case for other WAs currently described by multi-zone, constant density models. In addition, our work demonstrates that the TITAN code is well adapted to the study of the WA in active galactic nuclei, opening new prospects for the use of TITAN by a larger community.
\end{abstract}

Key words. galaxies: active - galaxies: quasars: individual: NGC 3783 - X-rays: general

\section{Introduction}

Many Active Galactic Nuclei (AGN) exhibit important X-ray absorption features caused by the presence of highly ionized gas located in the line-of-sight of the central continuum; this material is called "Warm Absorber" (hereafter WA). The first observations of WA gas in AGN were reported by Halpern et al. (1984) in the Einstein Observatory spectrum of MR 2251-178, a quasar displaying a large absorption feature around $1 \mathrm{keV}$; such a feature is consistent with the presence of gas photoionized by the hard X-rays produced near the AGN central engine.

Early ASCA observations have revealed the presence of intrinsic absorption in $\sim 50 \%$ of type 1 Seyferts. With the advent of space X-ray observatories such as Chandra and XMM-Newton, which carry aboard high-resolution grating spectrographs, an important set of high quality data became available; evidence for the presence of warm gas was found in many other types of AGN, e.g. Seyfert 2s (Kinkhabwala et al. 2002), Narrow Line Seyfert 1s (e.g. Matsumoto et al. 2004), BAL QSOs (Guainazzi et al. 2001; Grupe et al. 2003), and even some BL Lacs (e.g. Sambruna et al. 1997), although opinions diverge for these objects (Blustin 2004). In Seyfert 1s, the WA spectra revealed the presence of tens of absorption lines, covering a wide range of ionization states and blueshifted by a few hundreds to thousands of $\mathrm{km} \mathrm{s}^{-1}$ (an indication that the absorbing material is outflowing). In type $2 \mathrm{AGN}$, the data have shown the presence of emission lines.

Despite the undeniable improvements in our knowledge of the WA, some important issues remain a subject of debate, namely: (i) the geometry (multi-zone gas? clumps or flow?) and location of the WA; (ii) the physical conditions of the absorbing/emitting gas; and (iii) the importance of the WA in the energetics of the AGN. Trying to solve these questions requires not only high quality observations, but also an adequate modelling of the X-ray data through the use of reliable photoionization codes, which calculate the full radiative transfer and allow for a good physical description of the ionized gas.

The WA gas appears to be stratified, displaying multiple zones of different density, temperature, and ionization (e.g. Krolik \& Kriss 2001; Różańska et al. 2006). Until now, the WA in AGN has been modelled assuming the presence of several regions, each region being modelled in constant density and having a different ionization parameter ${ }^{1}\left(\xi\right.$, in units of erg $\left.\mathrm{cm} \mathrm{s}^{-1}\right)$,

1 The ionization parameter is defined as $L / n_{\mathrm{H}} R^{2}$, where $L$ is the source's bolometric luminosity, $n_{\mathrm{H}}$ is the hydrogen number density at the illuminated face of the medium, and $R$ is the distance from the WA to the illuminating source. 
column density $\left(N_{\mathrm{H}}\right.$, in units of $\left.\mathrm{cm}^{-2}\right)$, etc. However, a stratified medium can be obtained naturally, if one assumes the gas to be in pressure equilibrium. Such a medium could explain the presence of lines from different ionization states and account naturally for the other properties of a model composite of several constant density regions.

We have addressed the above-mentioned points through the study of the WA in NGC 3783, for which unrivaled quality Chandra/HETGS data (Kaspi et al. 2002) are available in the archives. There is a short discussion of the WA in NGC 3783 in the next section. We have modelled the data using the photoionization code TITAN, briefly described in Sect. 3. Our first results are given in Sect. 4, and the conclusions in Sect. 5.

\section{The Warm Absorber in NGC 3783}

NGC 3783 is a bright $(V \sim 13.5)$, nearby $(z=0.0097)$, Seyfert 1.5 galaxy observed in the Optical, UV and X-rays. The WA in this object has been discussed by several authors (e.g. Kaspi et al. 2001, 2002; Netzer et al. 2003; Krongold et al. 2003; Behar et al. 2003) based on Chandra and XMM-Newton data. These studies seem to agree on the presence of a multi-zone gas constituted by three or more regions of different ionizations. In terms of the kinematics of the WA, two or more velocity systems have been identified in Chandra observations; they are compatible with those observed in UV spectra. A single outflow velocity system $\left(v_{\text {flow }} \sim 600-800 \mathrm{~km} \mathrm{~s}^{-1}\right.$ ) seems enough to describe XMM-Newton observations, which have lower spectral resolution. There is no consensus on a possible correlation between the velocity shifts, or the $F W H M$ s, with the ionization potentials of the ions.

Although the WA in NGC 3783 has been the object of many studies, these have assumed either a constant density (e.g. Netzer et al. 2003), or a dynamical state (Chelouche \& Netzer 2005) for the modelling. In addition, they all require multiple zones of different density, temperature, and ionization, which are invoked to explain the large span in ionization observed in the WA spectrum. Furthermore, when plotted on the S-curve of thermal equilibrium $\log (T)$ vs. $\log (\xi / T)$ (where $T$ is the temperature of the medium and $\xi$ is the ionization parameter), these zones lie on a vertical line of roughly the same gas pressure, suggesting that a single medium in pressure equilibrium could account for the WA in this object. A physical modelling of the gas through adequate photoionization codes remains necessary to confirm or to invalidate such an assumption, that being the main goal of this paper.

We note that, for relatively thin slabs, the radiation and gas pressure remain constant, the temperature does not vary much within the slab, and constant pressure models can thus be approximated by a constant density model. However, for thicker media, the ratio between the gas and the radiation pressure varies across the slab, inducing variations in the density and the temperature. In such cases, it is important to take the total (gas+radiation) pressure into account.

\section{Modelling the WA with the TITAN code}

We have used the photoionization code TITAN, developed by our team (Dumont et al. 2000, 2002; Collin et al. 2004), to model the 900 ks Chandra/HETGS observations and to constrain the physical conditions of the WA gas in NGC 3783.

TITAN is well-suited both for the study of optically thick (Thomson thickness up to several tens) and thin media, such as the WA. It computes the gas structure in thermal and ionization equilibria, giving as output the ionization and temperature structures, as well as the reflected, emitted outward, and absorbed spectra. One advantage of TITAN is that it treats the transfer of both the lines and the continuum using the ALI (Accelerated Lambda Iteration) method, which very precisely computes line and continuum fluxes. Our line transfer treatment leads to a longer computation time than with other approximate methods; we thus use fewer lines than those available in other codes. Our atomic data include $\sim 10^{3}$ lines from ions and atoms of $\mathrm{H}, \mathrm{He}, \mathrm{C}, \mathrm{N}, \mathrm{O}, \mathrm{Ne}, \mathrm{Mg}, \mathrm{Si}, \mathrm{S}$, and Fe; they omit features like the Unresolved Transition Array (UTA) and the inner shell transitions (except the iron $\mathrm{K}$ lines). In the case where the radiation pressure and the losses from the lines are only a few tens percent of those from the continuum, as is the case in this study, the missing lines do not change the ionization and thermal structure much (Chevallier et al. 2006).

The total (gas + radiation) pressure is computed after the plasma temperature has been determined by the thermal balance equation. The pressure is kept fixed within the medium, thus determining the density profile. The model is iterated until convergence (see Dumont et al. 2000, 2002). In the presence of an external, strong, irradiating source, such as the hard $\mathrm{X}$-rays ionizing the WA material, the irradiated medium develops an ionization instability (Krolik et al. 1981). If the medium is in pressure equilibrium, it leads to a temperature discontinuity (e.g. Ko \& Kallman 1994) with at least three states of thermal equilibria (high-, intermediate-, and low-temperature), the middle one being unstable. Our approach to the thermal instability problem consists of adopting an equilibrium temperature value between the "hot" and the "cold" stable solutions. Such a method provides a unique solution (for a more complete discussion of the thermal instabilities with TITAN, see Sect. 4.1.3 in Różańska et al. 2002).

Our models assume a 1D plane-parallel geometry, with the WA medium being illuminated on one side by an incident continuum. We assume the incident radiation field to be concentrated in a very small pencil-like shape centered on the normal direction. The models are parameterized by the ionization parameter $\xi$, the column density $N_{\mathrm{H}}$, the hydrogen number density $n_{\mathrm{H}}$, and the incident flux Spectral Energy Distribution (SED). We can also consider a non-zero turbulent velocity $v_{\text {turb }}$.

Concerning NGC 3783 specifically, we note first that the turbulent velocity deduced from the observations (e.g. Kaspi et al. $2000)$ is highly supersonic compared to the thermal velocity $\left(v_{\text {th }}\right)$ in the absorbing medium. It is possible to address this situation either $(i)$ by taking into account the strong dissipation occuring through shock waves in the supersonic medium (this leads to a higher temperature than in a purely photoionized case); or (ii) by assuming a photoionized, inhomogeneous medium with several clumps lying in the line-of-sight. These gas clumps have an average relative velocity, that we identify as $v_{\text {turb }}$. We thus assume the WA in NGC 3783 to consist of such gas blobs embedded in a surrounding, hotter medium. The clumpy WA medium is assumed to be in total pressure equilibrium. We include the gas and the radiation pressure in the total pressure, but not the turbulent pressure resulting from taking a non-zero $v_{\text {turb }}$, since it is a quantity exterior to the clumps. One should, however, take the effects of $v_{\text {turb }}$ into account when computing the radiative transfer; this has been done for all our models.

The turbulent velocity acting on the line transfer is typically one order of magnitude larger than the thermal velocity of the heavy elements. The optical depth being inversely proportional to $v_{\text {turb }}$, this difference in velocity can be accounted for if each 


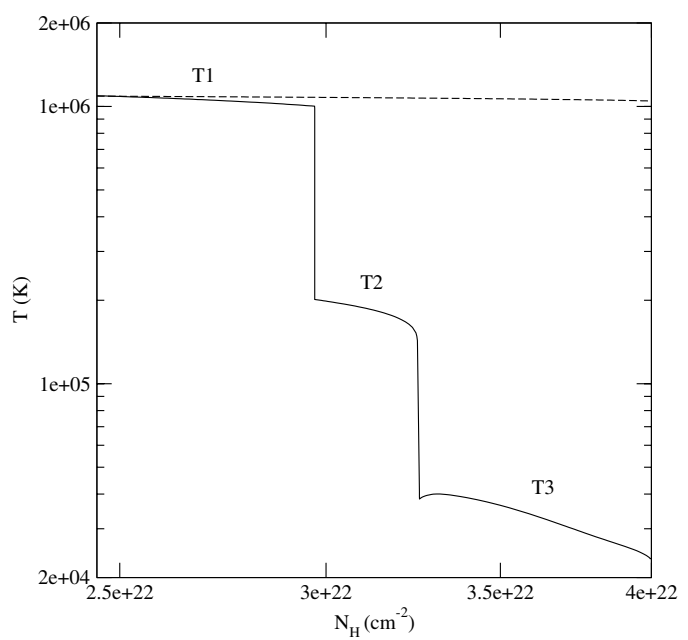

Fig. 1. The solid line represents the temperature profile resulting from our modelling of the WA as a single medium in total pressure equilibrium. In this case, the temperature discontinuities arise naturally. The dashed line corresponds to a single-zone WA with the same parameters, only modelled under constant density.

clump has a column density $\leq N_{\mathrm{H}} / n$ (where $N_{\mathrm{H}}$ corresponds to the column density of the clumpy system and $n$ is the number of clumps in the line-of-sight). From the observer's point of view, and for the purposes of our modelling, these clumps can be treated just like a continuous medium with column density $N_{\mathrm{H}}$, as each clump receives the radiation transmitted - and returned - by the other ones, while the hot surrounding medium does not have any effect on the radiation transfer.

We have computed a grid of 16 constant total pressure models to fit the WA in NGC 3783, covering the combinations of the 4 possible values of the ionization parameter $(2000<\xi<3500)$ and the total column density $\left(3 \times 10^{22}<N_{\mathrm{H}}<6 \times 10^{22}\right)$. The $n_{\mathrm{H}}$ was set to $10^{5} \mathrm{~cm}^{-3}$ (Netzer et al. 2003) and the $v_{\text {turb }}$ to $150 \mathrm{~km} \mathrm{~s}^{-1}$ (Kaspi et al. 2000). The incident SED used, given in Kaspi et al. (2001), covers the $0.2 \mathrm{eV}-400 \mathrm{keV}$ range. The models were computed using two different sets of abundances (Allen 1973; Netzer et al. 2003).

\section{Results and discussion}

Our study shows that the WA can be modelled under total pressure equilibrium conditions, and provides a best model with $\xi=2500 \mathrm{erg} \mathrm{cm} \mathrm{s}^{-1}, N_{\mathrm{H}}=4 \times 10^{22} \mathrm{~cm}^{-2}$, and cosmic (Allen 1973) abundances. While the results are stable for a $n_{\mathrm{H}}$ up to $10^{12} \mathrm{~cm}^{-3}$, the turbulent velocity of the clumpy medium seems to play a non-negligible role in the modelling. Our best model gives a reasonably good fit to the observed data, both for the continuum (following its overall shape up to $10 \mathrm{keV}$ and reproducing the $\mathrm{O}$ VII and $\mathrm{O}$ VIII edges) and the lines (both from high and low ionization). The observed and modelled spectra, as well as the corresponding $E W \mathrm{~s}$, will be presented in a forthcoming paper (Gonçalves et al., in preparation), in which the effects of using different abundances and $v_{\text {turb }}$ values will also be discussed.

For all the models in our grid, irradiation by the incident SED resulted in the formation of a hot surface zone, with an almost constant temperature, followed by a rapid drop by one order of magnitude at a certain depth, and a second temperature drop deeper in the slab. In Fig. 1 we give the temperature profiles for a constant density and a constant total pressure model, computed assuming the same parameters. We observe that, in the case of a constant density model, the temperature remains more or less constant along the medium. Thus, if a composite (i.e. multi-component) constant density model is assumed, as in Netzer et al. (2003), a relatively flat temperature profile is found for each component, with a different temperature value for each one. When a single medium in constant total pressure is assumed, the temperature drops arise naturally; such variations in temperature are accompanied by ionization fronts, resulting in different ionization fractions for the three sections (T1, T2, and T3) visible in the plot.

Our results can be compared to those of Netzer et al. (2003), who provide a detailed modelling of the WA in NGC 3783; these authors found three constant density components with different $\xi$ and $N_{\mathrm{H}}$ values. A full comparison between the multi-component model of Netzer et al. (as computed by TITAN) and our constant total pressure model will also be presented in a forthcoming paper. Figure 2 displays an example with the computed ionic column densities deduced from our best model and from the composite model of Netzer et al. vs. the "observed" ones determined through the curve of growth analysis in Netzer et al. (2003). The figure illustrates the good agreement between a single medium in constant total pressure and the three-zone model in constant density showing that, within the uncertainties, both models should lead to similar absorption spectra; this comparison used the same incident SED and abundances. Although Fig. 2 only shows 15 ions (for comparision with Netzer et al.), our model provides ionic column densitites for all the other ions in the atomic data, which include the lower ionization species that give rise to absorption lines in the UV.

Based on our best model results, and on the object's bolometric luminosity $\left(L \sim 2 \times 10^{44} \mathrm{erg} \mathrm{s}^{-1}\right)$ and black hole mass $\left(M_{\mathrm{BH}} \sim\right.$ $3 \times 10^{7} M_{\odot}$ ) (Peterson et al. 2004), we have calculated some quantities related to the WA. Assuming $n_{\mathrm{H}}=10^{5} \mathrm{~cm}^{-3}$, the sum of the thickness of all the clumps in the line-of-sight amounts to $\Delta R_{\mathrm{cl}} \sim 2 \times 10^{17} \mathrm{~cm}$ (a higher $n_{\mathrm{H}}$ would imply a smaller $\left.\Delta R_{\mathrm{cl}}\right)$. Knowing $\Delta R_{\mathrm{cl}}$, and to keep $\dot{M}_{\text {out }} / \dot{M}_{\text {Edd }} \leq 1$ (where $\dot{M}_{\text {out }}$ and $\dot{M}_{\text {Edd }}$ are the outflow and Eddington mass accretion rates), the volumic factor $f_{\text {vol }}$ should be $\leq 0.5$ (see Eq. (4) in Chevallier et al. 2006). As $f_{\text {vol }}$ corresponds to the size ratio of the "clumpy" to "whole WA" media $\left(\Delta R_{\mathrm{cl}} / \Delta R_{\mathrm{WA}}\right)$, we can estimate $\Delta R_{\mathrm{WA}}$ as being $\geq 4 \times 10^{17} \mathrm{~cm}$. Based on the previously given definition of $\xi$, we calculate the distance to the WA to be $R \leq 10^{18} \mathrm{~cm}$ (for $n_{\mathrm{H}}=10^{5} \mathrm{~cm}^{-3}$ ).

Given these values, the replenishment timescale $\left(R / v_{\text {flow }}\right)$ in NGC 3783 is of the order of $5 \times 10^{2} \mathrm{yr}$, and the dynamical timescale, several times higher. A satisfactory WA geometry could be obtained with $f_{\text {vol }} \sim 0.2$ and $n \geq v_{\text {turb }} / v_{\text {th }} \sim 10$. The geometrical thickness of a single clump should thus be $\leq 2 \times$ $10^{16} \mathrm{~cm}$, resulting in a sound crossing time of only $\sim 50 \mathrm{yr}$; if $n>10$, this time will be reduced in proportion. In such a picture, the replenishment and dynamical times are larger than the sound crossing time in each clump. In consequence, the clumps can adjust promptly to any change in the pressure equilibrium of the whole system. We note that these timescales are still much longer than the variation time of the illuminating flux; it could thus be objected that the gas in NGC 3783 cannot reach a state where it is in total pressure equilibrium with the illuminating source. We suggest that, unless the flux variations are very large, the medium would adopt a "quasi-pressure equilibrium" corresponding to an incident flux averaged over a long time. The flux and spectral variations should thus induce changes in the temperature and ionization equilibrium, but the density structure would stay about the same. The way the spectrum would be modified will be discussed in a forthcoming paper. 

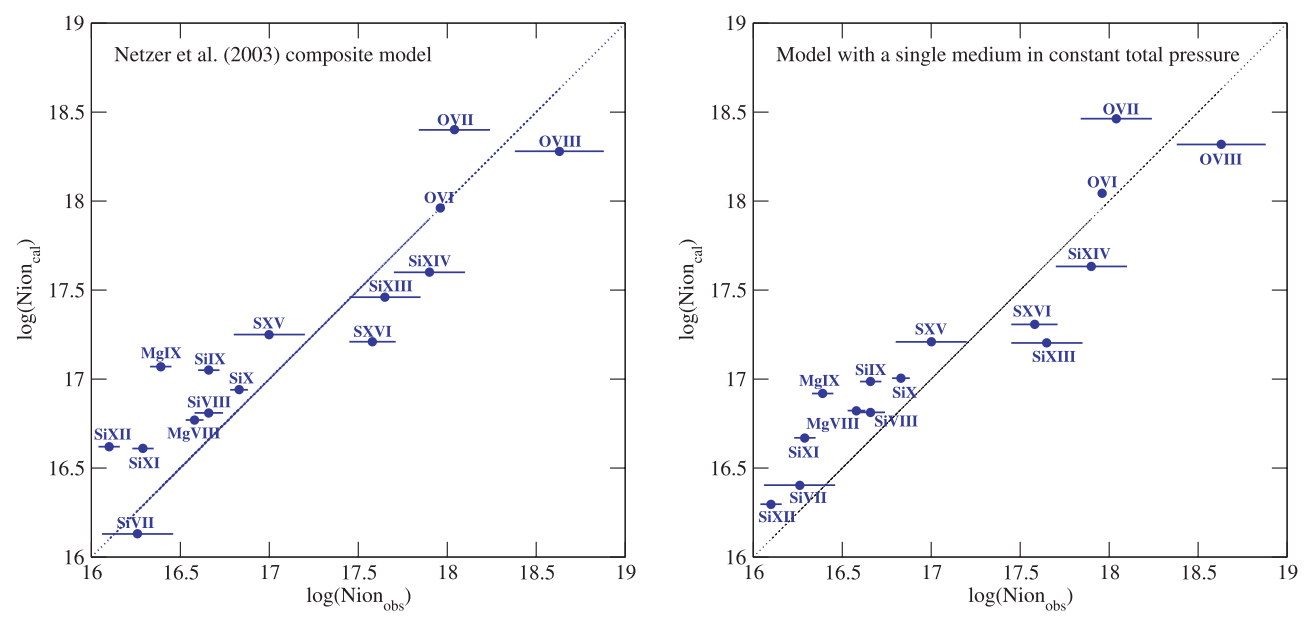

Fig. 2. Computed ionic column densities (in $\mathrm{cm}^{-2}$ ) for the Netzer et al. (2003) composite constant density model (left panel) and for our constant total pressure model (right panel) vs. the "observed" column densities determined through a curve of growth analysis. The observed O VI column density value corresponds to a lower limit, only. It was therefore replaced by the Netzer et al. computed value, which explains the absence of an error bar for this ion. The dotted line shows the diagonal on which the calculated values equal the observations.

\section{Conclusions}

1. We have shown that the WA in NGC 3783 can be modelled by a single medium in total pressure equilibrium.

2. Our grid of models has provided a best result for $\xi=$ $2500 \mathrm{erg} \mathrm{cm} \mathrm{s}^{-1}, N_{\mathrm{H}}=4 \times 10^{22} \mathrm{~cm}^{-2}$, and cosmic abundances.

3. Our work opens new prospects for the future use of the TITAN code by the community through larger grids of constant total pressure models to be made available via XSPEC and/or via Virtual Observatory facilities.

Acknowledgements. We acknowledge grant BPD/11641/2002 of the FCT, Portugal; grant 2P03D00322 of the PSCSR, Poland; support from LEA and astro-PF, Poland-France, and HBS, Germany.

\section{References}

Allen, C. W. 1973, Astrophysical quantities (U. London: Athlone Press) Behar, E., Rasmussen, A. P., Blustin, A. J., et al. 2003, ApJ, 598, 232

Blustin, A. J. 2004, Ph.D. Thesis, University College London, UK
Chelouche, D., \& Netzer, H. 2005, ApJ, 625, 95 Chevallier, L., Collin, S., Dumont, A.-M., et al. 2006, A\&A, 449, 493 Collin, S., Dumont, A.-M., \& Godet, O. 2004, A\&A, 419, 877 Dumont, A.-M., Abrassart, A., \& Collin, S. 2000, A\&A, 357, 823 Dumont, A.-M., Czerny, B., Collin, S., \& Zycki, P. T. 2002, A\&A, 387, 63 Gonçalves, A. C., et al., in preparation

Grupe, D., Mathur, S., \& Elvis, M. 2003, AJ, 126, 1159

Guainazzi, M., Marshall, W., \& Parmar, A. N. 2001, MNRAS, 323, 75 Halpern, J. P. 1984, ApJ, 281, 90

Kaspi, S., Brandt, W. N., Netzer, H., et al. 2000, ApJ, 535, L17 Kaspi, S., Brandt, W. N., Netzer, H., et al. 2001, ApJ, 554, 216

Kaspi, S., Brandt, W. N., George, I. M., et al. 2002, ApJ, 574, 643 Kinkhabwala, A., Sako, M., Behar, E., et al. 2002, ApJ, 575, 732 Ko, Y.-K., \& Kallman, T. R. 1994, ApJ, 431, 273 Krolik, J. H., \& Kriss, G. A. 2001, ApJ, 561, 684

Krolik, J. H., McKee, C. F., \& Tarter, C. B. 1981, ApJ, 249, 422

Krongold, Y., Nicastro, F., Brickhouse, N. S., et al. 2003, ApJ, 597, 832 Matsumoto, C., Leighly, K. M., \& Marshall, H. L. 2004, ApJ, 603, 456 Netzer, H., Kaspi, S., Behar, E., et al. 2003, ApJ, 599, 933 Peterson, B. M., Ferrarese, L., Gilbert, K. M., et al. 2004, ApJ, 613, 682 Różańska, A., Czerny, B., Dumont, A.-M., \& Collin, S. 2002, MNRAS, 332, 799 Różańska, A., Goosmann. R., Dumont. A.-M., \& Czerny, B. 2006, A\&A, in press [arXiv: astro-ph/0512310]

Sambruna, R. M., George, I. M., Madejski, G., et al. 1997, ApJ, 483, 774 